\title{
Magnetic Force Sensing Using a Self-Assembled Nanowire
}

\author{
N. Rossi, ${ }^{\dagger}$ B. Gross, ${ }^{\dagger}$ F. Dirnberger, ${ }^{\dagger}$ D. Bougeard, ${ }^{\dagger}$ and M. Poggio $*{ }^{\dagger}$ \\ ${ }^{\dagger}$ Department of Physics, University of Basel, 4056 Basel, Switzerland \\ ${ }^{\ddagger}$ Institut für Experimentelle und Angewandte Physik, Universität Regensburg, D-93040 Regensburg, Germany
}

Supporting Information

ABSTRACT: We present a scanning magnetic force sensor based on an individual magnet-tipped GaAs nanowire (NW) grown by molecular beam epitaxy. Its magnetic tip consists of a final segment of singlecrystal MnAs formed by sequential crystallization of the liquid Ga catalyst droplet. We characterize the mechanical and magnetic properties of such NWs by measuring their flexural mechanical response in an applied magnetic field. Comparison with numerical simulations allows the identification of their equilibrium magnetization configurations, which in some cases include magnetic vortices. To determine a NW's performance as a magnetic scanning probe, we measure its response to the field profile of a lithographically patterned

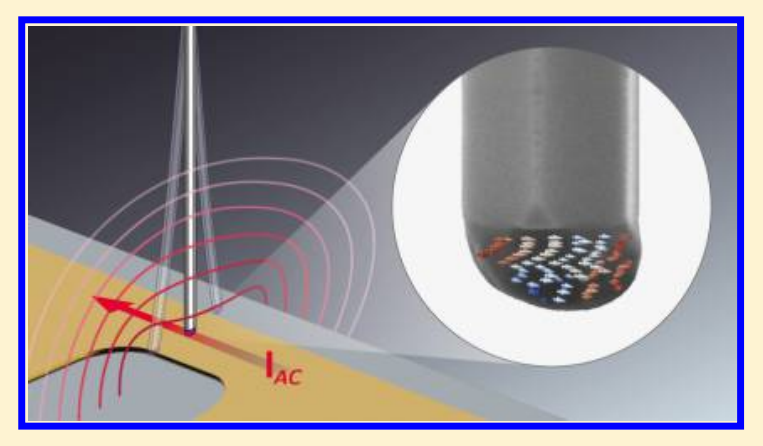
current-carrying wire. The NWs' tiny tips and their high force sensitivity make them promising for imaging weak magnetic field patterns on the nanometer-scale, as required for mapping mesoscopic transport and spin textures or in nanometer-scale magnetic resonance.

KEYWORDS: Nanowire, magnetic force microscopy, nanomagnetism, torque magnetometry, MnAs nanocrystal, sequential crystallization

A key component in any force microscopy is the force sensor. This device consists of a mechanical transducer, used to convert force into displacement, and an optical or electrical displacement detector. In magnetic force microscopy (MFM), mass-produced "top-down" Si cantilevers with sharp tips coated by a magnetic material have been the standard for years. Under ideal conditions, state-of-the-art MFM can reach spatial resolutions down to $10 \mathrm{~nm},{ }^{1}$ though more typically around $100 \mathrm{~nm}$. These conventional cantilevers are well-suited for the measurement of the large forces and force gradients produced by strongly magnetized samples.

The advent of nanostructures such as nanowires (NWs) and carbon nanotubes (CNTs) grown by "bottom-up" techniques has given researchers access to much smaller mechanical force transducers than ever before. This reduction in size implies both a better force sensitivity ${ }^{2}$ (see Supporting Information) and potentially a finer spatial resolution. ${ }^{3}$ Sensitivity to small forces provides the ability to detect weak magnetic fields and therefore to image subtle magnetic patterns; tiny concentrated magnetic tips have the potential to achieve nanometer-scale spatial resolution, while also reducing the invasiveness of the tip on the sample under investigation. Such improvements are crucial for imaging nanometer-scale magnetization textures such as domain walls, vortices and skyrmions, ${ }^{4-7}$ superconducting vortices, ${ }^{8,9}$ mesoscopic transport in two-dimensional systems, ${ }^{10}$ and small ensembles of nuclear spins. ${ }^{11-14}$

Recent experiments have demonstrated the use of single NWs and CNTs as sensitive scanning force sensors. ${ }^{15-18}$ When clamped on one end and arranged in the pendulum geometry, that is, with their long axes perpendicular to the sample surface to prevent snapping into contact, they probe both the size and direction of weak tip-sample forces. NWs have been demonstrated to maintain excellent force sensitivities around $1 \mathrm{aN} / \sqrt{\mathrm{Hz}}$ near sample surfaces $(<100$ $\mathrm{nm})$, due to extremely low noncontact friction. ${ }^{19}$ As a result, NW sensors have been used as transducers in force-detected nanometer-scale magnetic resonance imaging ${ }^{20}$ and in the measurement of optical and electrical forces. ${ }^{15-17}$ Nevertheless, the integration of a magnetic tip onto a NW transducer, and therefore the demonstration of NW MFM, has presented a significant practical challenge.

Here, we demonstrate such MFM transducers using individual GaAs NWs with integrated single-crystal MnAs tips, grown by molecular beam epitaxy (MBE). By monitoring each NW's flexural motion in an applied magnetic field, we measure its mechanical and magnetic properties. We determine the equilibrium magnetization configurations of each tip by comparing its magnetic response with micromagnetic simulations. In order to establish the sensitivity and resolution of the NWs as MFM transducers, we use them as scanning probes in the pendulum geometry. By analyzing their response to the magnetic field produced by a lithographically patterned current-carrying wire, we find that the MnAs tips can be approximated as nearly perfect magnetic dipoles. The thermally limited sensitivity of a typical NW to magnetic

Received: October 17, 2018

Revised: December 2, 2018

Published: January 2, 2019 

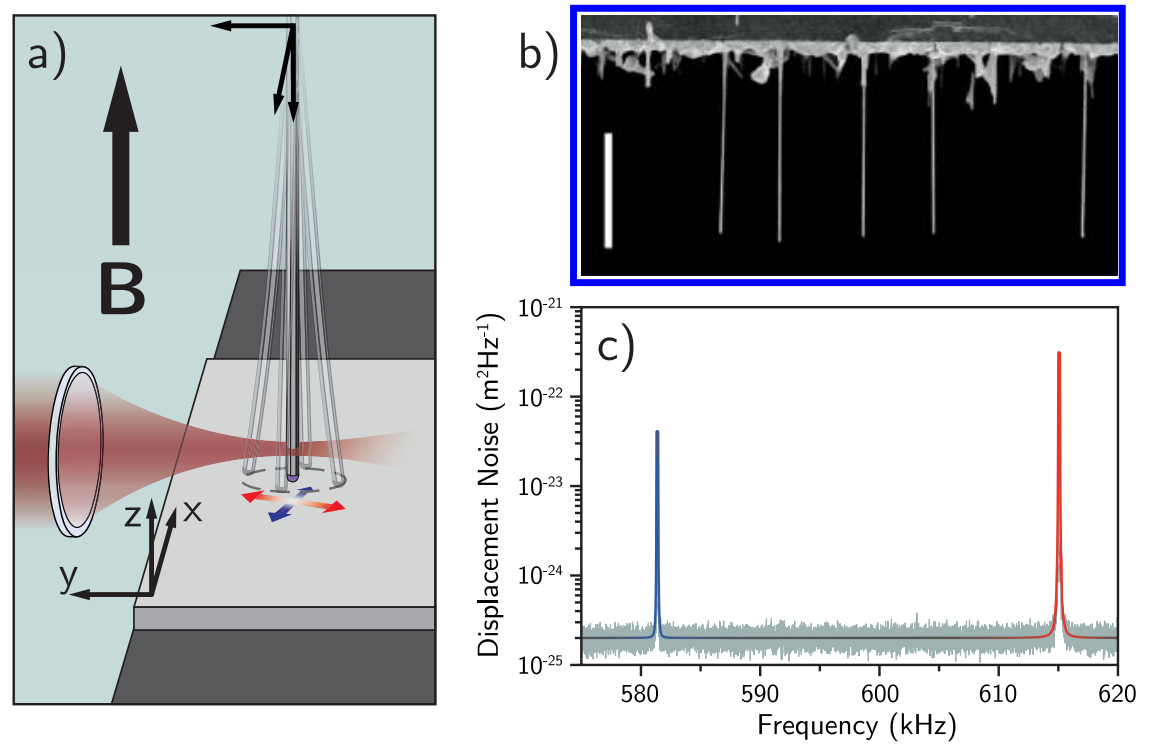

Figure 1. (a) Schematic drawing of the measurement setup. The NW is positioned in the waist of the focused laser beam by means of piezoelectric positioners. A second stack of positioners is used to approach and scan the sample of interest with respect to the NW. (b) Scanning electron micrograph (SEM) of the measured chip cleaved from the growth wafer with a $10 \mu \mathrm{m}$ scale-bar. (c) Displacement noise PSD of the first order flexural modes of a NW at $B=0 \mathrm{~T}$. Measured data (gray) and fit (colored line) representing the sum of two Lorentzians and white noise background $S_{\mathrm{N}}=2 \times 10^{-25} \mathrm{~m}^{2} / \mathrm{Hz}$. Such fits yield the orientation of each mode with respect to $\hat{\mathrm{y}}: \phi_{1}=+70^{\circ}, \phi_{2}=-20^{\circ}$ and the modes' effective mass as $780 \mathrm{fg}$, which is close to $790 \mathrm{fg}$ expected for a GaAs cylinder of density $5.32 \mathrm{~g} / \mathrm{cm}^{3}$ with hexagonal cross-section of maximal diameter 234 $\mathrm{nm}$ and length $16.8 \mu \mathrm{m}$. The extracted resonance frequency, spring constant, and intrinsic quality factor of the first (second) mode are $f_{0_{1}}=581$ $\mathrm{kHz}, k_{1}=10.5 \mathrm{mN} / \mathrm{m}$, and $Q_{1}=44650\left(f_{0_{2}}=615 \mathrm{kHz}, k_{2}=11.6 \mathrm{mN} / \mathrm{m}\right.$, and $\left.Q_{2}=48456\right)$.

field gradients is found to be $11 \mathrm{mT} /(\mathrm{m} \sqrt{\mathrm{Hz}})$, which corresponds to the gradient produced by $63 \mathrm{nA} / \sqrt{\mathrm{Hz}}$ through the wire at a tip-sample spacing of $250 \mathrm{~nm}$.

The GaAs NWs are grown on a $\mathrm{Si}(111)$ substrate by MBE using a self-catalyzed Ga-assisted growth method. ${ }^{21} \mathrm{~A}$ substrate temperature of $600{ }^{\circ} \mathrm{C}$ allows the growth of high quality crystalline NWs, which are typically $17 \pm 1 \mu \mathrm{m}$ long with a hexagonal cross-section of $225 \pm 15 \mathrm{~nm}$ in maximal diameter. In order to terminate the growth with a magnetic tip, the liquid Ga catalyst droplet at the top of the NW is heavily alloyed by a Mn flux. Then, to initiate its crystallization, it is exposed to an As background pressure for $30 \mathrm{~min}$. Under such conditions, the droplet undergoes a sequential precipitation: first, the Ga is preferentially consumed to build pure GaAs; next, the remaining $\mathrm{Mn}$ crystallizes in the form of MnAs. It has been shown by high-resolution transmission electron microscopy that this growth process leads to the formation of a welldefined hexagonal $\alpha$-MnAs wurzite crystal at the tip of a predominantly wurzite GaAs NW with an epitaxial relationship $[0001] \mathrm{MnAs} \|[0001] \mathrm{GaAs}$ along the $\mathrm{NW}$-axis. ${ }^{22}$ As reported for bulk MnAs, the tip is in a hexagonal ferromagnetic $\alpha$-phase up to the Curie temperature of about $313 \mathrm{~K}$, above which it undergoes a structural phase transition into an orthorhombic paramagnetic $\beta$-phase. ${ }^{23}$ MnAs crystals are characterized by a strong magnetocrystalline anisotropy with $\mathrm{K}=-1 \times 10^{6} \mathrm{~J} \mathrm{~m}^{-3}$ along the $c$-axis (hard axis). ${ }^{24}$ As a result, the magnetization of the tip will tend to lie in the plane (easy plane) orthogonal to the $c$-axis, which, in general, is coincident with the NW growth direction $\hat{\mathbf{n}}$.

The sample chip is cleaved directly from the $\mathrm{Si}$ wafer used for the NWs' growth. Using a micromanipulator under an optical microscope, we remove excess NWs to leave a single row of isolated and vertically standing NWs in proximity of the cleaved edge (Figure 1b). The chip is then loaded into a custom-built scanning probe microscope, which includes piezoelectric positioners to align a single $\mathrm{NW}$ within the focus of a fiber-coupled optical interferometer used to detect its mechanical motion. ${ }^{25}$ A second set of piezoelectric positioners enables the approach and scanning of the NW transducer over a sample of interest, as shown schematically in Figure 1a. The microscope is enclosed in a high-vacuum chamber at a pressure of $10^{-7} \mathrm{mbar}$ and inserted in the bore of a superconducting magnet at the bottom of a liquid ${ }^{4} \mathrm{He}$ bath cryostat. All the data presented here have been measured at a temperature $T=4.2 \mathrm{~K}$. In order to characterize the magnetic properties of the NWs, we apply a magnetic field $\mathbf{B}$ up to $\pm 8 \mathrm{~T}$ approximately parallel to $\hat{\mathbf{n}}$.

For the purposes of this work, we restrict our attention to the two fundamental flexural eigenmodes of the NWs, which oscillate along orthogonal directions, $\hat{\mathbf{r}}_{1}$ and $\hat{\mathbf{r}}_{2}$, and are shown schematically in Figure 1a. The coupling between the NW and the thermal bath results in a Langevin force equally driving both mechanical modes. Figure 1c shows a calibrated power spectral density (PSD) of the NW displacement noise, where the two resonance peaks correspond to the two orthogonally polarized modes. Such a measurement shows the projection of the modes' $2 \mathrm{D}$ thermal motion along the measurement axis $\hat{\mathbf{y}}$ (see Methods). Typical resonance frequencies range from 500 to $700 \mathrm{kHz}$ with quality factors between $2 \times 10^{4}$ and $5 \times 10^{4}$. For each NW, the doublet modes are completely separated by a frequency splitting $\delta$ of several hundred times the peak line width. In fact, it has been shown that even very small $(<1 \%)$ cross-sectional or clamping asymmetries can split the modes by several line widths. ${ }^{26}$ Nevertheless, the quality factors of the doublet modes differ by less than $1 \%$. The spring constants extracted from fits to the PSD for each flexural mode are on the order of $10 \mathrm{mN} / \mathrm{m}$, yielding a mechanical dissipation 
(friction coefficient) and a thermally limited force sensitivity down to $50 \mathrm{pg} / \mathrm{s}$ and few aN $/ \sqrt{\mathrm{Hz}}$, respectively.

We exploit this high mechanical sensitivity to probe the magnetization of each individual magnetic tip. As in dynamic cantilever magnetometry (DCM), ${ }^{27-29}$ we can extract magnetic properties of each MnAs tip from the mechanical response of the NW to a uniform external magnetic field $\mathbf{B}$. In such a field, the resonance frequency of each orthogonal flexural mode $f_{i}(i=1,2)$ is modified by the curvature of the system's magnetic energy $E_{\mathrm{m}}$ with respect to rotations $\theta_{i}$, corresponding to each mode's oscillation. For $\theta_{i} \ll 1, r_{i}=l_{\mathrm{e}} \theta_{i}$, where $l_{\mathrm{e}}$ is an effective length, which takes into account the shape of the flexural mode. ${ }^{29}$ The resulting frequency shift $\Delta f_{i}$ $=f_{i}-f_{0_{i}}$, where $f_{0_{i}}$ is the resonance frequency at $B=0$, is given by $^{30,31}$

$$
\Delta f_{i}=\frac{f_{0_{i}}}{2 k_{i} l_{\mathrm{e}}^{2}}\left(\left.\frac{\partial^{2} E_{\mathrm{m}}}{\partial \theta_{i}^{2}}\right|_{\theta_{i}=0}\right)
$$

where $k_{i}$ is each mode's spring constant.

We perform measurements of $\Delta f_{i}(B)$ on several NWs by recording the thermal displacement PSD of their doublet modes as a function of $B$. For nearly all investigated NWs (11 out of 12), $\Delta f_{i}(B)$ is negative for all applied fields (e.g., Figure $2 \mathrm{a}$ and Figure $3 \mathrm{a})$. In general, negative values of $\Delta f_{i}$

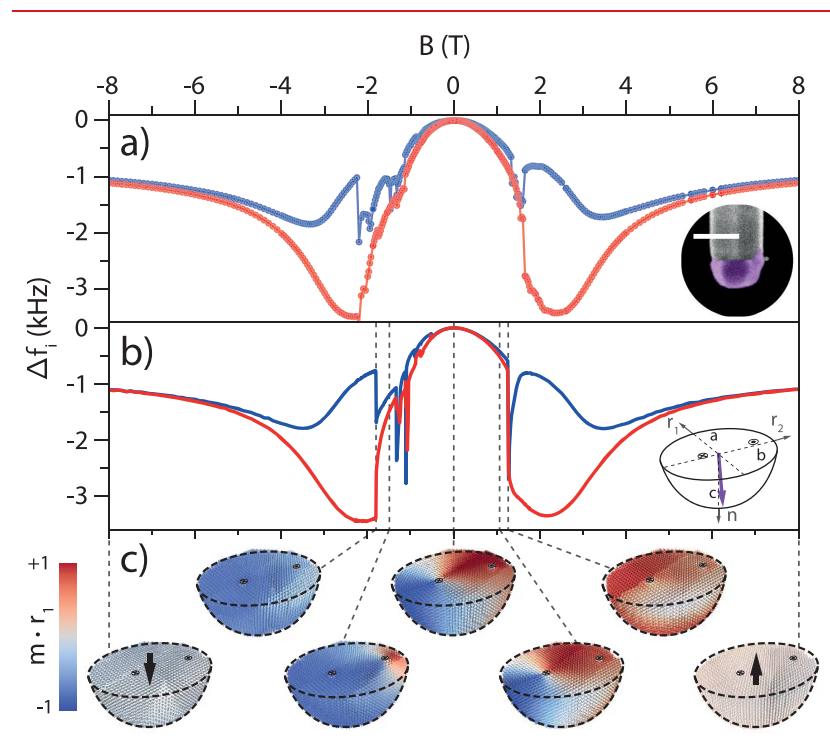

Figure 2. DCM measurement of NW1 revealing the existence of a magnetic vortex in the MnAs tip. (a) Plot of the $\Delta f_{i}(B)$ for $i=1(i=$ 2) in blue (red), extracted at each $B$ from the thermal displacement PSD. $f_{0_{1}}=613 \mathrm{kHz}, f_{0_{2}}=636 \mathrm{kHz}$, and $k_{1}=7.7 \mathrm{mN} / \mathrm{m}, k_{2}=8.3 \mathrm{mN} /$ m. $B$ is stepped from positive to negative field values. Inset: false-color SEM of NW1's MnAs tip with a $200 \mathrm{~nm}$ scale-bar. (b) Plot of the corresponding $\Delta f_{i}(B)$ simulated for a half-ellipsoid. Inset: schematic diagram of the half-ellipsoid geometry used to approximate the MnAs tip, where $a=85 \mathrm{~nm}, b=90 \mathrm{~nm}, c=95 \mathrm{~nm}$. The simulation uses parameters for $\mathrm{MnAs}$ from the literature and a hard axis (purple arrow) given by $\theta_{\mathrm{K}}=4.2^{\circ}$ and $\phi_{\mathrm{K}}=45.5^{\circ}$. Black dots and crosses indicate the position and direction of the sites of pinned magnetization. (c) Simulated magnetization configurations for $B$ indicated by the dashed lines. Each cone, associated with a discretized volume, is color-coded with the magnitude of the magnetization component along $\hat{\mathbf{r}}_{1}$. Large black arrows indicate the net magnetization for $B= \pm 8$ $\mathrm{T}$.
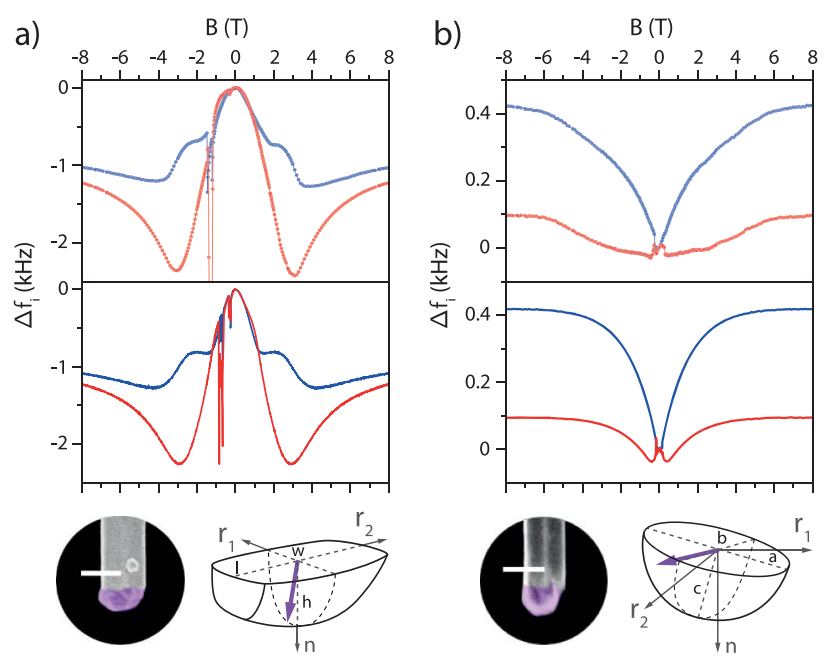

Figure 3. DCM measurements of (a) NW2 and (b) NW3. The upper plots show the measurements of $\Delta f_{i}(B)$ and the lower plots show the corresponding simulations for $i=1(i=2)$ in blue (red). $B$ is stepped from positive to negative field values. At the bottom, SEMs of the MnAs tips in false color (scale-bar $200 \mathrm{~nm}$ ) along with a sketch of the tip geometry used in the simulation. (a) For NW2, the frequency shifts are calculated by using the mechanical characteristics reported in Figure 1c. The hard axis (purple arrow) is set to $\theta_{\mathrm{K}}=8^{\circ}$ and $\phi_{\mathrm{K}}=$ $-9.5^{\circ}$. The asymmetric geometry is a truncated ellipsoid of length $l=$ $230 \mathrm{~nm}$, width $w=115 \mathrm{~nm}$, and depth $h=110 \mathrm{~nm}$. (b) For NW3, resonance frequencies and spring constants are $f_{0_{1}}=435 \mathrm{kHz}, f_{0_{2}}=$ $447 \mathrm{kHz}$, and $k_{1}=14.3 \mathrm{mN} / \mathrm{m}, k_{2}=15 \mathrm{mN} / \mathrm{m}$, respectively. The hard axis (purple arrow) is nearly perpendicular to $\hat{\mathbf{n}}$ with $\theta_{\mathrm{K}}=68.3^{\circ}$ and $\phi_{\mathrm{K}}=142.2^{\circ}$. The tip is approximated as an elongated ellipsoid with $a$ $=b=85 \mathrm{~nm}$ and $c=90 \mathrm{~nm}$ and it is tilted with respect to the modes' reference system, so that its semiaxis $c$ is tilted by $\theta_{\mathrm{t}}=20^{\circ}$ and $\phi_{\mathrm{t}}=$ $210^{\circ}$ with respect to $\hat{\mathbf{n}}$.

correspond to a local maximum in $E_{\mathrm{m}}\left(\theta_{i}\right)$ with respect to $\theta_{i}$. This behavior is consistent with $\mathbf{B}$ being aligned along the magnetic hard axis of the MnAs tip, which should be along the NW growth-axis. In Figure 2a, NW1 shows a particularly ideal magnetic response, in which the high-field frequency shift of both modes asymptotically approaches the same negative value. This behavior indicates a MnAs particle with a hard axis along $\hat{\mathbf{n}}$ and no preferred easy axis in the $r_{1} r_{2}$-plane.

In order to gain a deeper understanding of the DCM signal, we carry out simulations of the MnAs tips using Mumax3, ${ }^{32}$ which employs the Landau-Lifshitz-Gilbert micromagnetic formalism using finite-difference discretization. For each value of $B$, the simulations determine the equilibrium magnetization configuration of the $\mathrm{MnAs}$ particle and the corresponding values of $\Delta f_{i}$ (see Methods and Supporting Information). The geometry of the MnAs tip is estimated by SEM and set within the simulation with respect to $\hat{\mathbf{r}}_{1}, \hat{\mathbf{r}}_{2}$, and $\hat{\mathbf{n}}$.

The DCM response of the MnAs tip measured in Figure 2a and shown in the inset is simulated by approximating its shape as a half ellipsoid with dimensions given in the inset of Figure $2 \mathrm{~b}$ and its caption. The excellent agreement between the measured and simulated $\Delta f_{i}(B)$, plotted in Figure $2 \mathrm{a}, \mathrm{b}$, respectively, allows us to precisely determine the direction of the magnetic hard axis. As expected, this axis is found to be nearly along $\hat{\mathbf{n}}$ : just $\theta_{\mathrm{K}}=2.5^{\circ}$ away from $\hat{\mathbf{n}}$ and $\phi_{\mathrm{K}}=19.5^{\circ}$ from $\hat{\mathbf{r}}_{1}$. Furthermore, as shown in Figure $2 c$, the simulations relate a specific magnetization configuration to each value of $B$. In this particular case, a stable vortex configuration in the easy plane is seen to enter (exit) from the edge in correspondence with the 

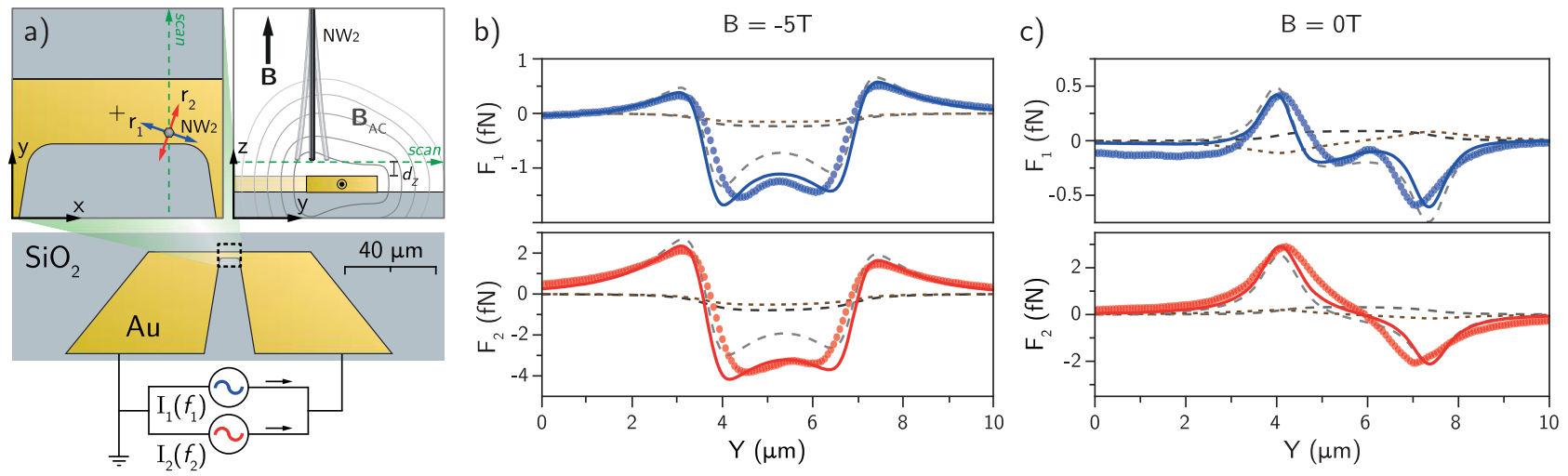

Figure 4. Characterization of NW as magnetic scanning probe. (a) Schematic drawing of the bottom sample consisting of a $3 \mu \mathrm{m}$ wide, $6 \mu \mathrm{m}$ long, and $240 \mathrm{~nm}$ thick $\mathrm{Au}$ wire on an insulating $\mathrm{SiO}_{2}$ substrate. NW2 is approached at a tip-sample spacing $d_{z}=250 \mathrm{~nm}$ and scanned across the constriction. (b,c) Plots of the measured (dotted line) and calculated (solid line) forces $F_{i}$ driving the first (blue) and the second (red) mode over a $10 \mu \mathrm{m}$ long line scan. For each plot, three distinct drive contributions are shown as dashed lines: the monopole (black), dipole (gray), and torque (brown) terms. The simulated response is fit by setting $q_{0}=-1 \times 10^{-10} \mathrm{~A} \cdot \mathrm{m},|\mathrm{m}|=8 \times 10^{-16} \mathrm{~A} \cdot \mathrm{m}^{2}$ with $\theta_{\mathrm{m}}=2^{\circ}, \phi_{\mathrm{m}}=20^{\circ}$ for the high magnetic field case (b) and $q_{0}=4 \times 10^{-11} \mathrm{~A} \cdot \mathrm{m},|\mathrm{m}|=5.3 \times 10^{-16} \mathrm{~A} \cdot \mathrm{m}^{2}$ with $\theta_{\mathrm{m}}=87^{\circ}, \phi_{\mathrm{m}}=42^{\circ}$ for the zero field case (c).

abrupt discontinuities in the eigenmodes' frequencies around $+2 \mathrm{~T}(-2 \mathrm{~T})$. Between these two fields, the vortex core moves from one side to the other, inducing several discontinuities in $\Delta f_{i}(B)$. The smoothness of the measured frequency shifts around $B=0 \mathrm{~T}$ indicates pinning of the vortex and is wellreproduced in the simulation by the introduction of two sites of pinned magnetization (see Supporting Information).

Most measured NWs (10 out of 12) present DCM curves as shown by NW2 in Figure 3a. Despite the similarity of these curves to those shown in Figure 2a, no sharp discontinuity is observed upon sweeping $B$ down from saturation (forward applied field). Furthermore, the high-field frequency shift of both modes does not asymptotically approach the same negative value as in Figure $2 \mathrm{a}$. Both of these effects can be explained by taking into account magnetic shape anisotropy in the MnAs tips. Despite the nearly perfect symmetry of NW1's tip, most of the crystallized MnAs droplets are asymmetric in the $r_{1} r_{2}$-plane. This asymmetry introduces an effective magnetic easy axis in the $r_{1} r_{2}$-plane. In fact, the measured $\Delta f_{i}(B)$ shown in Figure 3a are well-reproduced by a simulation that takes into a account the geometry of NW2's MnAs tip as observed by SEM. While small refinements in the microscopic geometry, which often cannot be confirmed by the SEM, affect how well the simulation matches every detail of the measured $\Delta f_{i}(b)$ (see Supporting Information), the precise orientation of the hard axis and the direction of the effective shape anisotropy in the $r_{1} r_{2}$-plane sensitively determine the curves' overall features (e.g., their high field asymptotes and shape).

In general, simulations show that shape anisotropy restricts the field range for a stable magnetic vortex to reverse applied field. In small forward applied field and in remanence, the magnetization evolves through a configuration with a net magnetic dipole in the $r_{1} r_{2}$-plane. Only upon application of a reverse field, does this configuration smoothly transform into a vortex, resulting, for NW2, in a subtle dip in $\Delta f_{i}(B)$ around $B=$ $-0.3 \mathrm{~T}$. At a reverse field close to $B=-2 \mathrm{~T}$, an abrupt jump indicates the vortex's exit and the appearance of a singledomain state, which eventually turns toward B. This analysis indicates that NW2's tip, as well as the majority of the MnAs tips, present a dipole-like remanent configuration pointing in the $r_{1} r_{2}$-plane, rather than vortex-like configuration with a core pointing along $\hat{\mathbf{n}}$, as in NW1. Such remanent magnetic dipoles have been already observed by MFM in similar tips. ${ }^{22,33}$

In rare cases ( 1 of 12$)$, such as the one reported for NW3 in Figure $3 \mathrm{~b}$, we measure mostly positive $\Delta f_{i}(B)$ with different high-field asymptotes for each eigenmode. This behavior indicates a MnAs particle, whose hard axis points approximately in the $r_{1} r_{2}$-plane. In fact, the features of the measured $\Delta f_{i}(B)$ in Figure $2 \mathrm{~b}$ are reproduced by a simulation considering a nearly symmetric half-ellipsoid with a hard-axis lying $\theta_{\mathrm{K}}=68.3^{\circ}$ from $\hat{\mathbf{n}}$ and $\phi_{\mathrm{K}}=142.2^{\circ}$ from $\hat{\mathbf{r}}_{\mathbf{1}}$. These data are clear evidence that crystallization of the liquid droplet can occasionally occur along a direction far off from the NW growth axis.

In order to test the behavior of these NWs as scanning magnetic sensors, we approach a typical one (NW2) to a current-carrying $\mathrm{Au}$ wire patterned on a $\mathrm{SiO}_{2}$ substrate, as described in Figure 4a. Once in the vicinity of the wire constriction, the NW's two modes are excited by the Biot-Savart field $\mathbf{B}_{\mathrm{AC}}$ resulting from an oscillating drive current $I=I_{1} \sin \left(2 \pi f_{1} t\right)+I_{2} \sin \left(2 \pi f_{2} t\right)$, where $I_{1}=I_{2}=50 \mu \mathrm{A}$. Single $10 \mu \mathrm{m}$ long line scans are acquired by moving the NW across the wire at the fixed tip-sample spacing $d_{z}=250 \mathrm{~nm}$. This spacing is chosen to match the scanning probe's tip size, because smaller spacings would not improve its spatial resolution. Both the resonant frequencies $f_{i}$ and displacement amplitudes $r_{i}$ are tracked using two phase-locked loops and the corresponding values of the force driving each mode at resonance are then calculated as $F_{i}=r_{i} k_{i} / Q_{i}$ (see Supporting Information).

Using an approach similar to that used to calibrate MFM tips, ${ }^{34-36}$ we model the force exerted by a well-known magnetic field profile on the magnetic tip by using the socalled point-probe approximation. This approximation models the complex magnetization distribution of the tip as an effective monopole moment $q_{0}$ and a dipole moment $\mathbf{m}$ located at a distance $d$ from the tip apex (the monopole contribution compensates for the non-negligible spatial extent of the tip). The magnetic force acting on each mode is then given by $D_{i}=$ $q_{0} \mathbf{B}_{\mathrm{AC}} \cdot \hat{\mathbf{r}}_{i}+\nabla\left(\mathbf{m} \cdot \mathbf{B}_{\mathrm{AC}}\right) \cdot \hat{\mathbf{r}}_{i}$. Moreover, we also consider the magnetic torque $\boldsymbol{\tau}=\mathbf{m} \times \mathbf{B}_{\mathrm{AC}}$ generated at the tip, which results in a torsion and/or bending of the NW depending on its orientation. Although this contribution is negligible in 
conventional MFM, the NW modes' short effective length $\left(l_{\mathrm{e}}=\right.$ $12.2 \mu \mathrm{m})$ and soft spring constant $\left(k_{1} \approx k_{2} \approx 11 \mathrm{mN} / \mathrm{m}\right)$, make the bending component of the torque responsible for an observable displacement along $\hat{\mathbf{r}}_{i}$. We then model the total force driving each mode as $F_{i}=D_{i}+T_{i}$, where $T_{i}=\left(l_{\mathrm{e}}^{-1} \hat{\mathbf{n}} \times \boldsymbol{\tau}\right)$. $\hat{\mathbf{r}}_{i}$. $d$ is set to $100 \mathrm{~nm}$ from the tip (i.e., approximately at the base of the MnAs crystallite) for the best fits, while $q_{0}$ and $\mathbf{m}$ are used as free parameters. The precise spatial dependence of the field $\mathbf{B}_{\mathrm{AC}}$ produced by the current $I$ is calculated using the finite-element package COMSOL (see Supporting Information).

We characterize the NW's magnetic response at $B=-5 \mathrm{~T}$ and $B=0$. In the high field case shown in Figure $4 b$, a fit of the two driving forces is obtained with an effective dipole $\mathbf{m}$ nearly along B with a magnitude $|\mathrm{m}|=0.45 M_{\mathrm{s}} V$, where $M_{\mathrm{s}}=1.005 \mathrm{~T}$ is the saturation magnetization of $\mathrm{MnAs}^{37}$ and $V=2.24 \times$ $10^{-21} \mathrm{~m}^{3}$ is the volume of the tip defined in the magnetometry simulation. In general, the estimation of $V$ from SEM is approximate due to the difficulty in determining the precise three-dimensional geometry and in distinguishing regions of nonmagnetic material inside the tip or at its surface. The fit returns a small, but nonzero $q_{0}$ and shows a very good agreement with the measured forces sensed by the two modes. In Figure 4c, the same line scan is performed in absence of external field and, as expected, the observed response changes radically, because the magnetization lies mostly in the easy plane and is not completely saturated. In the fit, the effective dipole contribution is dominant and mostly orthogonal to the NW axis $\hat{\mathbf{n}}$ with $|\mathbf{m}|=0.3 M_{s} V$. The direction of $\mathbf{m}$ is closely related to the torque contribution making the fit particularly sensitive to the value of $\phi_{\mathrm{m}}$. On the basis of both numerical simulations and control experiments, using the applied magnetic field to initialize the MnAs tip magnetization along opposing directions we find spurious electrostatic driving of the NW modes to be negligible.

The NWs' high force sensitivity combined with highly concentrated and strongly magnetized dipole-like tips give them an exquisite sensitivity to magnetic field gradients. In order to quantify this sensitivity, we restrict our attention to the second mode (red) of NW2, positioning it at the point of maximal response over the wire at $d_{z}=250 \mathrm{~nm}$ and $B=0 \mathrm{~T}$ (i.e., $y=4.5 \mu \mathrm{m}$ on Figure $4 \mathrm{c}$ and roughly at the position of the NW in Figure 4a). The displacement signal $r_{2}$ is measured with a lock-in amplifier while decreasing the driving current $I=I_{2}$ $\sin \left(2 \pi f_{2} t\right)$. The sweeps plotted in Figure $5 \mathrm{a}, \mathrm{b}$ show the expected linear response as well as a wide dynamic range. In Figure $5 b$, we focus on the low-current regime, showing both the in-phase $X$ (signal + noise) and quadrature $Y$ (noise) response. By simple linear regression, we extract the signal as $\bar{X}=\beta I_{2} / \sqrt{2}$ with a transduction factor $\beta=0.26 \mathrm{~nm} / \mu \mathrm{A}$. The noise in both $X$ and $Y$ is found to be Gaussian and fully ascribable to the NW's thermal motion with variances $\sigma_{X}^{2} \approx \sigma_{Y}^{2}$ $\approx S_{r_{2}}\left(\omega_{2}\right) \times \mathrm{BW}_{\text {neq }}$, where $S_{r_{2}}(\omega)$ is a fit to the second mode's thermal PSD shown in Figure $5 c, \omega_{2}$ is the resonant angular frequency of the second mode, and $\mathrm{BW}_{\text {neq }}$ is the lock-in's equivalent noise bandwidth. As shown in Figure $5 \mathrm{c}$ for $Y$, the mode's thermal PSD is assumed constant around its value at resonance $S_{r_{2}}\left(\omega_{2}\right)=4 k_{\mathrm{B}} T \frac{Q_{2}}{k_{2} \omega_{2}}$, due to the narrow measurement bandwidth. Therefore, the NW's second mode has a thermally limited displacement sensitivity of $\sqrt{S_{r_{2}}\left(\omega_{2}\right)}=16 \mathrm{pm} / \sqrt{\mathrm{Hz}}$, equivalent to a force sensitvity of
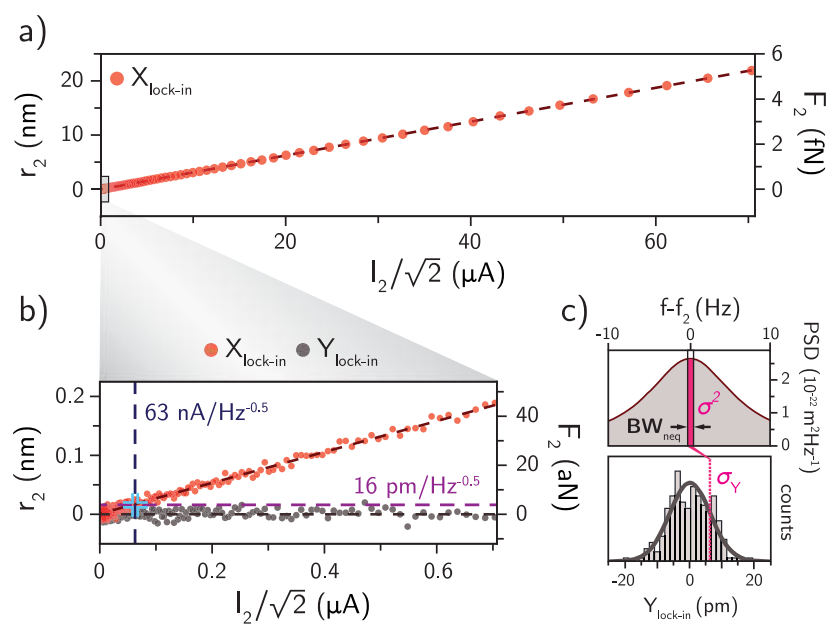

Figure 5. NW sensitivity to a resonant current drive at a distance of $250 \mathrm{~nm}$. (a) Plot of the oscillation amplitude $r_{2}$ for each value of the current amplitude $I_{2}$, both quantities are root mean squared. (b) Plot of in-phase response to the drive $(X)$ and quadrature signal $(Y)$ for a finer current sweep from $I_{2}=1 \mu \mathrm{A}$ to $I_{2}=1 \mathrm{nA}$. The lock-in demodulator low-pass filter noise equivalent bandwidth was set to $\mathrm{BW}_{\text {neq }}=0.156 \mathrm{~Hz}$ and each point was averaged for $2.5 \mathrm{~s}$. Both signals are linearly fit (dark dashed lines). The intersection (light blue cross) between the linearly fit signal $\bar{X}$ and the displacement sensitivity (dashed purple line), shows a current sensitivity of $63 \mathrm{nA} / \sqrt{\mathrm{Hz}}$ (dashed blue line). (c) Noise analysis on the quadrature channel Y. On top, a close-up of the second mode's PSD fit and the noise power $\sigma^{2}=4.2 \times 10^{-23} \mathrm{~m}^{2}$ within the measurement bandwidth $\mathrm{BW}_{\text {neq. }}$. Below, the histogram of the noise measured on quadrature channel $(Y)$ in $(\mathrm{b})$, fitted by a Gaussian $\mathrm{N}\left(0, \sigma_{Y}\right)$ with $\sigma_{Y}=6.5 \mathrm{pm} \sim \sqrt{\sigma^{2}}$, confirming the thermally limited nature of the sensitivity measurement.

$\sqrt{S_{r_{2}}\left(\omega_{2}\right)} k_{2} / Q_{2}=4 \mathrm{aN} / \sqrt{\mathrm{Hz}}$. Given the measured current transduction factor $\beta$ at the tip-sample spacing $d_{z}=250 \mathrm{~nm}$, we obtain a sensitivity to current flowing through the wire of $63 \mathrm{nA} / \sqrt{\mathrm{Hz}}$.

Such sensitivity to electrical current compares favorably to that of other microscopies capable of imaging current through Biot-Savart fields, including scanning Hall microscopy, magneto-optic microscopy, scanning SQUID microscopy, microwave impedance microscopy, and scanning nitrogen-vacancy magnetometry. ${ }^{38,39}$ Because of the dipole-like character of the MnAs tip, this transduction of current into displacement is dominated by the effect of the time-varying magnetic field gradient generated by the current: $F_{i} \approx \nabla\left(\mathbf{m} \cdot \mathbf{B}_{\mathrm{AC}}\right) \cdot \hat{\mathbf{r}}_{i}=$ $\mathbf{m} \cdot \nabla\left(\mathbf{B}_{\mathrm{AC}} \cdot \hat{\mathbf{r}}_{i}\right)$. Although the torque resulting from the timevarying magnetic field produces an effective force, $T_{i}$, as seen in Figure $4 b, c$, this term is typically secondary. Therefore, from COMSOL simulations of the field produced by current flowing through the wire, we find this current sensitivity to correspond to a sensitivity to magnetic field gradient of $11 \mathrm{mT} /(\mathrm{m} \sqrt{\mathrm{Hz}})$ at the position of the tip's effective point probe, i.e., $d_{z}+d=350$ $\mathrm{nm}$ above the surface. The direction of this gradient sensitivity depends on the direction of the mode used. Having quantified the NW's response to magnetic field gradients, we can calculate its sensitivity to other magnetic field sources, including a magnetic moment (dipole field), a superconducting vortex (monopole field), or an infinitely long and thin line of current. $^{38}$ In particular, we expect a moment sensitivity of $54 \mu_{\mathrm{B}} / \sqrt{\mathrm{Hz}}$, a flux sensitivity of $1.3 \mu \Phi_{0} / \sqrt{\mathrm{Hz}}$, and line- 
current sensitivity of $9 \mathrm{nA} / \sqrt{\mathrm{Hz}}$. These values show the capability of magnet-tipped NWs as probes of weak magnetic field patterns and the huge potential for improvement if tips sizes and tip-sample spacings can be reduced (see Supporting Information). Smaller magnetic tips may be produced using optimized growth processes or focused ion beam milling. Alternatively, the production of different types of magnettipped NWs could be attempted through the evaporation of magnetic caps on sharp nonmagnetic $\mathrm{NWs}^{40}$ or by direct focused ion beam-induced deposition.

In addition to improved sensitivity, NW MFM provides other potential advantages compared to conventional MFM. First, scanning in the pendulum geometry with the NW oscillating in the plane of the sample has the characteristics of lateral MFM. This technique, which is realized with the torsional mode of a conventional cantilever, distinguishes itself from the more commonly used tapping-mode MFM in its ability to produce magnetic images devoid of spurious topography-related contrast and in a demonstrated improvement in lateral spatial resolution of up to $15 \%{ }^{41}$ Second, the nanometer-scale magnetic particle at the apex of the NW force sensor minimizes the size of the MFM tip, allowing for optimal spatial resolution and minimal perturbation of the investigated sample.

The prospect of increased sensitivity and resolution, combined with few restrictions on operating temperature, make NW MFM ideally suited to investigate nanometer-scale spin textures, skyrmions, superconducting and magnetic vortices, as well as ensembles of electronic or nuclear spins. High force sensitivity coupled with small tip size could allow magnetic NW sensors to work both close to a sample, maximizing spatial resolution, and in a regime of weak interaction, remaining noninvasive. Such magnetic tips may also open opportunities to study current flow in 2D materials and topological insulators. The ability of a NW sensor to map all in-plane spatial force derivatives ${ }^{16,17}$ should provide fine detail of stray field profiles above magnetic and current carrying samples, in turn providing detailed information on the underlying phenomena. Directional measurements of dissipation may also prove useful for visualizing domain walls and other regions of inhomogeneous magnetization. As shown by Grutter et al., dissipation contrast, which maps the energy transfer between the tip and the sample, strongly depends on the sample's nanometer-scale magnetic structure. ${ }^{42}$

Methods. Interferometric Detection. The linearly polarized light emitted by a laser diode with wavelength $\lambda=1553$ $\mathrm{nm}$ is directly coupled to a polarization maintaining optical fiber, sent through the 5\% transmission arm of a 95:5 fiberoptic coupler, collimated and focused on the NW by a pair of lenses. This confocal reflection microscopy setup, analogous to the one described in ref 43 , focuses light to a minimum beam waist of $w_{0}=1.65 \mu \mathrm{m}$ (see Supporting Information). The light incident on the NW has a power of $25 \mu \mathrm{W}$ and is polarized along its long axis. Light scattered back by the NW interferes with light reflected by the fiber's cleaved end, resulting in a low-finesse Fabry-Perot interferometer. A fast photoreceiver monitors variations in the intensity of reflected light, allowing for the sensitive detection of NW motion. The interferometric signal is proportional to the projection of the NW's motion along the direction of the interference pattern's gradient in the $x y$-plane. The magnitude of this gradient at the position of the NW determines the interferometer's transduction factor. By positioning the NW within the optical waist or changing the wavelength of the laser, it is possible to measure the motion projected along arbitrary directions (see Supporting Information). In this work, mechanical displacements are measured exclusively along $\hat{\mathbf{y}}$ by centering the $\mathrm{NW}$ on the optical axis. When the focal spot is aligned at the end of the NW, we observe heating up to $15 \mathrm{~K}$ in the displacement PSD of the two NW modes, due to absorption of light by the MnAs particle. By measuring the NW displacement several $\mu \mathrm{m}$ from the tip, negligible heating is observed.

Numerical Simulations. Micromagnetic simulations are carried out with Mumax3. We set the saturation magnetization $\mu_{0} M_{\mathrm{s}}=1.005 \mathrm{~T}$, the exchange stiffness constant $A=10 \mathrm{pJ} / \mathrm{m}$, and the magnetocrystalline anisotropy $K=-1.2 \times 10^{6} \mathrm{~J} / \mathrm{m}^{3}$ in correspondence with the values reported for $\mathrm{MnAs}$ in the literature. ${ }^{24,37}$ We model the geometry of each MnAs magnetic tip based on observations made by SEM. Space is discretized into cubic mesh elements, which are $5 \mathrm{~nm}$ on a side, corresponding to the dipolar exchange length of the material $l_{\mathrm{ex}}=\sqrt{2 A /\left(\mu_{0} M_{\mathrm{s}}^{2}\right)}$. The validity of this discretization is confirmed by comparing the results of a few representative simulations with simulations using much smaller mesh sizes. Mumax3 determines the equilibrium magnetization configuration for each external field value by numerically solving the Landau-Lifshitz-Gilbert equation. Because the microscopic processes in a MnAs tip are expected to be much faster than the NW resonance frequencies, the magnetization of the tip is assumed to be in its equilibrium orientation throughout the cantilever oscillation. The simulation also yields the total magnetic energy $E_{\mathrm{m}}$ corresponding to each configuration. We calculate $\Delta f_{i}$ in (1) by numerically evaluating the second derivatives of $E_{\mathrm{m}}$ with respect to $\theta_{i}$. At each field, we calculate $E_{\mathrm{m}}$ at the equilibrium angles $\theta_{i}=0$ and at small deviations from equilibrium $\theta_{i}= \pm \delta \theta_{i}$. For small $\delta \theta_{i}$, the second derivative can be approximated by a finite difference: $\left.\frac{\partial^{2} E_{\mathrm{m}}}{\partial \theta_{i}^{2}}\right|_{\theta_{i}=0} \approx \frac{E_{\mathrm{m}}\left(\delta \theta_{i}\right)-2 E_{\mathrm{m}}(0)+E_{\mathrm{m}}\left(-\delta \theta_{\mathrm{i}}\right)}{\left(\delta \theta_{i}\right)^{2}}$. By setting $f_{0_{i}}, k_{i}$, and $l_{\mathrm{e}}$ to their measured values, we then arrive at the $\Delta f_{i}$ corresponding to each magnetization configuration in the numerically calculated field dependence.

\section{ASSOCIATED CONTENT}

\section{S Supporting Information}

The Supporting Information is available free of charge on the ACS Publications website at DOI: 10.1021/acs.nanolett.8b04174.

How cantilever size affects force sensitivity; optical setup and detection details; displacement calibration; simulations of magnetic tips; simulation of $\mathbf{B}_{\mathrm{AC}}$ magnetic stray field; calculation of spin and flux sensitivity (PDF) Movie of simulated magnetization reversal in the MnAs tips of NW1 (AVI)

Movie of simulated magnetization reversal in the MnAs tips of NW2 (AVI)

Movie of simulated magnetization reversal in the MnAs tips of NW3 (AVI)

\section{AUTHOR INFORMATION}

\section{Corresponding Author}

*E-mail: martino.poggio@unibas.ch.

ORCID $\odot$

M. Poggio: 0000-0002-5327-051X 


\section{Notes}

The authors declare no competing financial interest.

\section{ACKNOWLEDGMENTS}

We thank Sascha Martin and his team in the machine shop of the Physics Department at the University of Basel for help building the measurement system. We thank Lorenzo Ceccarelli for help with magnetic field simulations and Prof. Ilaria Zardo for suggesting the collaboration. We acknowledge the support of the Kanton Aargau, the ERC through Starting Grant NWScan (Grant 334767), the SNF under Grant 200020-178863, the Swiss Nanoscience Institute, the NCCR Quantum Science and Technology (QSIT), and the DFG via project GR1640/5-2 in SPP 153.

\section{REFERENCES}

(1) Schmid, I.; Marioni, M. A.; Kappenberger, P.; Romer, S.; Parlinska-Wojtan, M.; Hug, H. J.; Hellwig, O.; Carey, M. J.; Fullerton, E. E. Phvs. Rev. Lett. 2010, 105, 197201.

(2) Poggio, M. Nat. Nanotechnol. 2013, 8, 482-483.

(3) Lisunova, Y.; Heidler, J.; Levkivskyi, I.; Gaponenko, I.; Weber, A.; Caillier, C.; Heyderman, L. J.; Kläui, M.; Paruch, P. Nanotechnology 2013, 24, 105705.

(4) Rondin, L.; Tetienne, J.-P.; Rohart, S.; Thiaville, A.; Hingant, T.; Spinicelli, P.; Roch, J.-F.; Jacques, V. Nat. Commun. 2013, 4, 2279.

(5) Tetienne, J.-P.; Hingant, T.; Kim, J.-V.; Diez, L. H.; Adam, J.-P.; Garcia, K.; Roch, J.-F.; Rohart, S.; Thiaville, A.; Ravelosona, D.; Jacques, V. Science 2014, 344, 1366-1369.

(6) Tetienne, J.-P.; et al. Nat. Commun. 2015, 6, 6733.

(7) Dovzhenko, Y.; Casola, F.; Schlotter, S.; Zhou, T. X.; Büttner, F.; Walsworth, R. L.; Beach, G. S. D.; Yacoby, A. 2016, arXiv:1611.00673 [cond-mat].

(8) Thiel, L.; Rohner, D.; Ganzhorn, M.; Appel, P.; Neu, E.; Müller, B.; Kleiner, R.; Koelle, D.; Maletinsky, P. Nat. Nanotechnol. 2016, 11, 677-681.

(9) Pelliccione, M.; Jenkins, A.; Ovartchaiyapong, P.; Reetz, C.; Emmanouilidou, E.; Ni, N.; Bleszynski Jayich, A. C. Nat. Nanotechnol. 2016, 11, 700-705.

(10) Tetienne, J.-P.; Dontschuk, N.; Broadway, D. A.; Stacey, A.; Simpson, D. A.; Hollenberg, L. C. L. Science Advances 2017, 3, e1602429.

(11) Rugar, D.; Mamin, H. J.; Sherwood, M. H.; Kim, M.; Rettner, C. T.; Ohno, K.; Awschalom, D. D. Nat. Nanotechnol. 2015, 10, 120124.

(12) Häberle, T.; Schmid-Lorch, D.; Reinhard, F.; Wrachtrup, J. Nat. Nanotechnol. 2015, 10, 125-128.

(13) Degen, C. L.; Poggio, M.; Mamin, H. J.; Rettner, C. T.; Rugar, D. Proc. Natl. Acad. Sci. U. S. A. 2009, 106, 1313-1317.

(14) Poggio, M.; Degen, C. Nanotechnology 2010, 21, No. 342001.

(15) Gloppe, A.; Verlot, P.; Dupont-Ferrier, E.; Siria, A.; Poncharal, P.; Bachelier, G.; Vincent, P.; Arcizet, O. Nat. Nanotechnol. 2014, 9, 920-926.

(16) Rossi, N.; Braakman, F. R.; Cadeddu, D.; Vasyukov, D.; Tütüncüoglu, G.; Fontcuberta i Morral, A.; Poggio, M. Nat. Nanotechnol. 2016, 12, 150-155.

(17) de Lépinay, L. M.; Pigeau, B.; Besga, B.; Vincent, P.; Poncharal, P.; Arcizet, O. Nat. Nanotechnol. 2016, 12, 156-162.

(18) Siria, A.; Niguès, A. Sci. Rep. 2017, 7, 11595.

(19) Nichol, J. M.; Hemesath, E. R.; Lauhon, L. J.; Budakian, R. Phys. Rev. B: Condens. Matter Mater. Phys. 2012, 85, No. 054414.

(20) Nichol, J. M.; Naibert, T. R.; Hemesath, E. R.; Lauhon, L. J.; Budakian, R. Phys. Rev. X 2013, 3, No. 031016.

(21) Colombo, C.; Spirkoska, D.; Frimmer, M.; Abstreiter, G.; Fontcuberta i Morral, A. Phys. Rev. B: Condens. Matter Mater. Phys. 2008, 77, 155326.
(22) Hubmann, J.; Bauer, B.; Körner, H. S.; Furthmeier, S.; Buchner, M.; Bayreuther, G.; Dirnberger, F.; Schuh, D.; Back, C. H.; Zweck, J.; Reiger, E.; Bougeard, D. Nano Lett. 2016, 16, 900-905.

(23) Bean, C. P.; Rodbell, D. S. Phvs. Rev. 1962, 126, 104-115.

(24) De Blois, R. W.; Rodbell, D. S. Phvs. Rev. 1963, 130, 13471360.

(25) Nichol, J. M.; Hemesath, E. R.; Lauhon, L. J.; Budakian, R. Appl. Phys. Lett. 2008, 93, 193110.

(26) Cadeddu, D.; Braakman, F. R.; Tütüncüoglu, G.; Matteini, F.; Rüffer, D.; Fontcuberta i Morral, A.; Poggio, M. Nano Lett. 2016, 16, 926-931.

(27) Rossel, C.; Bauer, P.; Zech, D.; Hofer, J.; Willemin, M.; Keller, H. J. Appl. Phvs. 1996, 79, 8166-8173.

(28) Harris, J. G. E.; Awschalom, D. D.; Matsukura, F.; Ohno, H.; Maranowski, K. D.; Gossard, A. C. Appl. Phvs. Lett. 1999, 75, 11401142.

(29) Stipe, B. C.; Mamin, H. J.; Stowe, T. D.; Kenny, T. W.; Rugar, D. Phvs. Rev. Lett. 2001, 86, 2874.

(30) Gross, B.; Weber, D. P.; Rüffer, D.; Buchter, A.; Heimbach, F.; Fontcuberta i Morral, A.; Grundler, D.; Poggio, M. Phys. Rev. B: Condens. Matter Mater. Phys. 2016, 93, No. 064409.

(31) Mehlin, A.; Gross, B.; Wyss, M.; Schefer, T.; Tütüncüoglu, G.; Heimbach, F.; Fontcuberta i Morral, A.; Grundler, D.; Poggio, M. Phys. Rev. B: Condens. Matter Mater. Phys. 2018, 97, 134422.

(32) Vansteenkiste, A.; Leliaert, J.; Dvornik, M.; Helsen, M.; GarciaSanchez, F.; Van Waeyenberge, B. AIP Adv. 2014, 4, 107133.

(33) Ramlan, D. G.; May, S. J.; Zheng, J.-G.; Allen, J. E.; Wessels, B. W.; Lauhon, L. I. Nano Lett. 2006, 6, 50-54.

(34) Lohau, J.; Kirsch, S.; Carl, A.; Dumpich, G.; Wassermann, E. F. I. Appl. Phvs. 1999, 86, 3410-3417.

(35) van Schendel, P. J. A.; Hug, H. J.; Stiefel, B.; Martin, S.; Güntherodt, H.-I. J. Appl. Phys. 2000, 88, 435-445.

(36) Kebe, T.; Carl, A. J. I. Appl. Phvs. 2004, 95, 775-792.

(37) Engel-Herbert, R.; Hesjedal, T.; Schaadt, D. M.; Däweritz, L.; Ploog, K. H. Appl. Phvs. Lett. 2006, 88, 052505.

(38) Kirtley, J. R. Rep. Prog. Phys. 2010, 73, 126501.

(39) Chang, K.; Eichler, A.; Rhensius, J.; Lorenzelli, L.; Degen, C. L. Nano Lett. 2017, 17, 2367-2373.

(40) Kim, W.; Dubrovskii, V. G.; Vukajlovic-Plestina, J.; Tütüncüoglu, G.; Francaviglia, L.; Güniat, L.; Potts, H.; Friedl, M.; Leran, J.-B.; Fontcuberta i Morral, A. Nano Lett. 2018, 18, 49-57.

(41) Kaidatzis, A.; García-Martín, J. M. Nanotechnology 2013, 24, 165704.

(42) Grütter, P.; Liu, Y.; LeBlanc, P.; Dürig, U. Appl. Phys. Lett. 1997, 71, 279-281.

(43) Högele, A.; Seidl, S.; Kroner, M.; Karrai, K.; Schulhauser, C.; Sqalli, O.; Scrimgeour, J.; Warburton, R. Rev. Sci. Instrum. 2008, 79, No. 023709. 